\title{
Infectious gastritis caused by a mixed fungal and bacterial infection
}

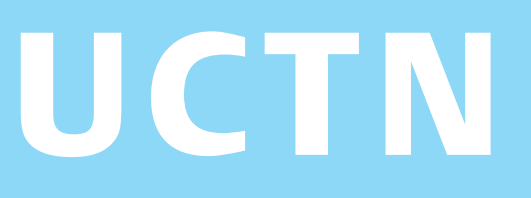

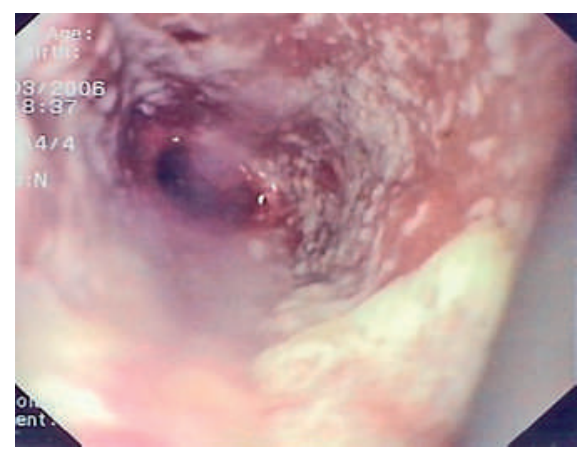

Figure 1 At esophagogastroduodenoscopy, the esophageal mucosa was seen to be covered by circumferential, white-gray, adherent plaques.

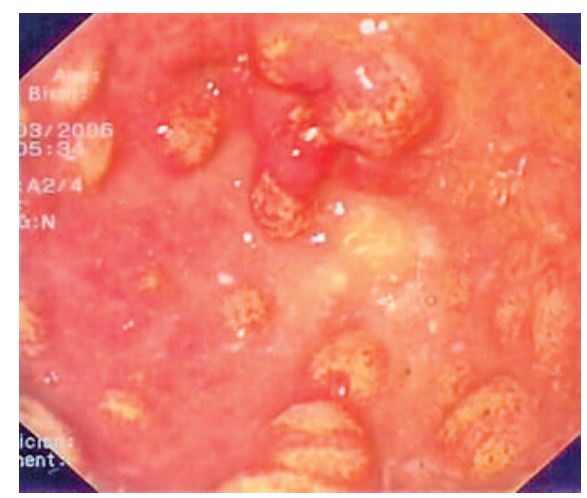

Figure 2 The antral mucosa had a nodular appearance and was covered by yellowish deposits.

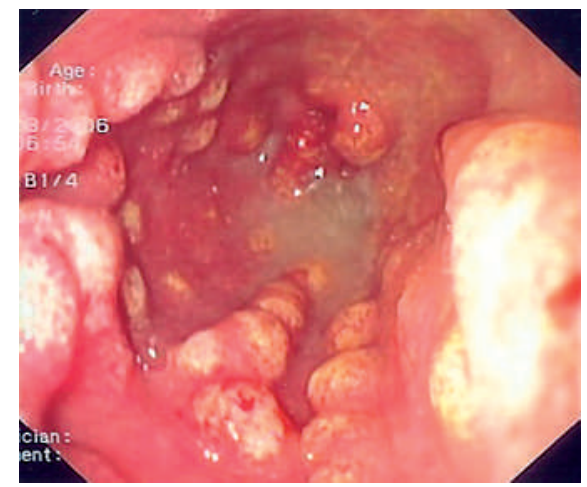

Figure 3 Nodular folds covered by yellowish debris were seen in the pyloric region.

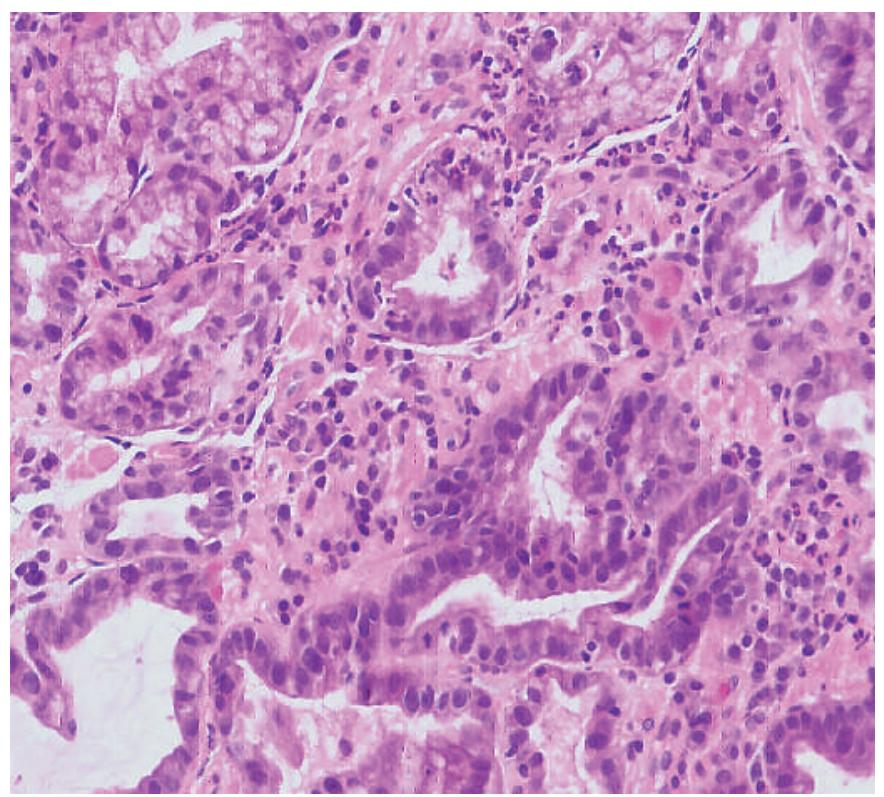

Figure 4 Histological examination showed the typical changes of acute gastritis, with a rich inflammatory infiltrate dominated by neutrophils.

A 76-year-old man presented with dysphagia and odynophagia. Five years earlier, he had undergone a partial gastroesophageal resection for adenocarcinoma of the esophagus. Over the past few years he had also required several dilations for pyloric stenosis (secondary to vagotomy). He had a history of chronic bronchitis with frequent exacerbations treated by short courses of antibiotics. An upper gastrointestinal endoscopy was performed. The esophageal mucosa was covered by circumferential, white-gray, adherent plaques (Figure $\mathbf{1}$ ). The antral mucosa had a nodular appearance and was covered by yellowish deposits (Figure 2 ), and nodular folds covered by yellowish debris were seen in the pyloric region (Figure 3 ). Histological examination revealed a picture of acute gastritis, with a rich inflammatory infiltrate dominated by neutrophils (Figure 4). Cultures yielded isolates of Escherichia coli, Candida albicans, and Candida glabrata. The patient responded well to antifungal and antibiotic treatment.

Fungal colonization in the stomach is common, and is associated with old age, malnutrition, diabetes, trauma, burns, surgery, total parenteral nutrition, intravascular or bladder catheterization, anti- secretory therapy, immunosuppressive treatment, and the use of wide-spectrum antibiotics [1,2]. Bacterial gastritis is a rare entity, however, and most reports describe only one or two cases, with a review of the literature [3]. Several conditions have been reported as predisposing to bacterial gastritis, including immunosuppression, upper respiratory and buccopharyngeal infections [4], gastric ischemia, and endoscopic therapy (mucosectomy and polypectomy) [5]. Our patient probably developed esophageal candidosis secondary to long-term antibiotic use. Gastric stasis secondary to vagotomy and the respiratory tract infections may also have played a role in the development of the $E$. coli infection of the gastric mucosa.

We emphasize that endoscopic appearances such as these should raise the suspicion of infectious gastritis and that multiple biopsies with bacteriological examination should be performed to confirm the diagnosis.

Endoscopy_UCTN_Code_CCL_1AB_2AD_3AC

DOI: 10.1055/s-2007-966089 
L. Negreanu' ${ }^{1}$, P. Assor ${ }^{1}$, F. Bumsel' ${ }^{2}$, E.-H. Metman ${ }^{1}$

${ }^{1}$ Department of Gastroenterology, Trousseau Hospital, François Rabelais University, Tours, France

${ }^{2}$ Department of Pathology, Trousseau Hospital, François Rabelais University, Tours, France.
References

${ }^{1}$ Scott BB, Jenkins D. Gastro-oesophageal candidiasis. Gut 1982; 23: 137-139

2 Savino JA, Agarwal N, Wry P et al. Routine prophylactic antifungal agents (clotrimazole, ketokonazole and nystatin) in nontransplant, nonburned, critically ill, surgical and trauma patients. J Trauma 1994; 36: 20-25

${ }^{3}$ Kim GY, Ward J, Henessey B et al. Phlegmonous gastritis: case report and review. Gastrointest Endosc 2005; 61: 168 - 174

${ }^{4}$ Schultz MJ, van der Hulst RW, Tytgat GN. Acute phlegmonous gastritis. Gastrointest Endosc 1996; 44: 80-83

${ }^{5}$ Lee BS, Kim SM, Seong JK et al. Phlegmonous gastritis after endoscopic mucosal resection. Endoscopy 2005; 37: 490-493
Corresponding Author

\section{Negreanu, M.D.}

Service d'Hépato-Gastroentérologie Hôpital Trousseau

Rue de Loches 37044 Tours Cedex

France

Fax: $\quad+33-247478428$

E-mail: negreanu_99@yahoo.com 